\title{
Understanding attitudes towards social media segregation: spatial metaphors in the discussion of Twitter blocklists
}

\author{
Dawn Wheatley \\ School of Communications, Dublin City University, Ireland \\ dawn.wheatley@dcu.ie, ORCID ID: 0000-0002-8751-4535
}

Eirik Vatnoey

Department of Linguistics and Scandinavian Studies, University of Oslo, Norway

eirik.vatnoy@iln.uio.no, ORCID ID: 0000-0003-2490-009X

\begin{abstract}
Blocking other users is a common act on Twitter but one which is underexplored from a scholarly perspective, particularly the analysis of mass blocklists. Although traditionally associated with harassment, blocklists are increasingly engaged to create individualised environments that align with users' personal convictions and exclude apparent transgressors. This study uses a pro-choice blocklist (Repeal Shield) created during the 2018 Irish abortion referendum campaign to explore how users interpret these altered boundaries and blocklists' influence on the Twitter landscape. A metaphor analysis of more than 2,000 tweets discussing the blocklist highlights the dominant concepts in how users visualise Twitter as both a personal space and a battlefield, in which mental health is a key factor. By drawing on discussions of spatiality, agency, gender and online interactions, we can see how these blocking affordances allow users to exist in spaces in which they construct their own parameters to feel safer, raising questions about how harm, health and risk are understood. The article explores how users make sense of conflicting images like 'safe spaces' or 'echo chambers', highlighting the apparent policing role held by blocklists. Users are negotiating the type of civic space in which they want to exist as norms of engagement versus avoidance collide; although digital spaces have always accommodated fragmented interests, the technological affordances of blocklists provide more rigid boundaries, highlighting how the evolving architecture of social media allows users to redefine the parameters of their own online spaces.
\end{abstract}

Keywords:

Blockbot; Blocklist; Social Media; Twitter; Spatial Metaphor; Metaphor Analysis

Please note: this is a post-print version of an article published in Information, Communication \& Society: https://doi.org/10.1080/1369118X.2020.1749696 


\section{Introduction}

In 2015, a headline on TheDailyBeast.com dramatically claimed that a social media blocklist 'could save the internet' (Poulos, 2015); shortly after, Wired.com warned readers to 'Beware the blocklist' (Auerbach, 2015). As with many aspects of digital culture, different affordances garner mixed responses, reflecting the spectrum of attitudes and expectations about how we engage - or disengage - with others online. Papacharissi has suggested that those studying online architectures should 'examine personal interpretations of the options provided, and analyse how individuals incorporate, reject or adapt' architectural elements (2009, p. 216); such an assessment serves as the starting point of this study, which looks at how a pro-choice blocklist, Repeal Shield, was conceptualised by Twitter users in Ireland in the run-up to the 2018 abortion referendum. Of particular interest is how users draw on physical and geographical spatial terms and expressions to understand the effect that a technical tool was having on a digital space, thus providing valuable insights into contemporary discussions around online segregation.

Blocklists, or blockbots, are a filtering tool hosted on third-party websites which can be linked directly to subscribers' Twitter accounts. Administrators curate a list of Twitter users, manually or algorithmically, typically grounded in some common theme/pattern in the blocked users’ behaviour. In this study, @Repeal_Shield said it was focused on pro-life 'trolls' and bot/sockpuppet accounts who the administrators felt were ruining the Twitter environment in the run-up to the abortion referendum. A previously popular blocklist, ggautoblock, was established amid the Gamergate controversy in 2014 as a response to women being targeted in the gaming community, and most existing literature highlights blocklists' association as an effective tool to combat harassment, helping users restore control and improve social media experiences, and facilitating counterpublic participation (Benesch, 2017; Geiger, 2016; Golbeck et al., 2017; Jhaver et al., 2018; Maréchal, 2016). However, concerns have been raised, with some internet users 
suggesting blocklists breach normative rules of online interaction as the interference with natural flows and exchanges undermines the discursive, democratic nature of spaces like Twitter, and that blocklists publicly discriminate against those listed (Gosse \& O’Meara, 2018). Twitter discussions around the presence of Repeal Shield have illustrated strong arguments in favour and against the blocklist, with division over the very principle of such lists, clear tension between individual rights versus broader societal expectations, alongside performative elements of in-group/out-group expressions (Wheatley \& Vatnoey 2020).

In this article, we explore the relationship between technological affordances and spatial understandings of social media. A systematic metaphor analysis (Schmitt, 2005) of discourse surrounding the blocklist reveals two dominant conceptual metaphors relating to how users talk and think about Twitter as a space. The first we label TWITTER AS A PERSONAL SPACE, as it reveals a conceptualisation of both Twitter and the blocklist through a series of metaphorical connections to the domestic and personal. The other we label TWITTER AS A BATTLEFIELD, as it reveals a similar connection to conflict or violent struggle as a source domain for metaphorical perceptions. We also identify two other central conceptual metaphors that, albeit not directly relating to spatial conceptualisations, are central to understanding the relationship between technological affordances and spatial understandings present in the material. The first is the conceptualisation of MENTAL HEALTH AS A RESOURCE that must be protected, defended, and used with care. This is closely connected to a psychosocial understanding of offensive utterances as a form of violence harming people's mental health. The second is the conceptual metaphor of BLOCKLISTS AS POLICING, in the sense of illegitimate monitoring and censoring of political opponents. At the core of this configuration of conceptual metaphors is the term 'safe space'. Although in no way confined to Twitter or social media, terms like 'safe space', and their contested meaning and value, provide an entry point into how people talk and think about 
changing digital landscapes and the reflective relationship between understandings of these landscapes and the practices they contain. The Repeal Shield case demonstrates how this configuration of conceptual metaphors steers how people talk and think about new affordances introduced to the media platform. Also, it demonstrates how the sometimes-conflicting conceptualisations of such affordances reveal and reinforce diverging understandings of the space in which they are introduced.

\section{Existing research}

\section{Understanding metaphors of space in the digital era}

The study of metaphors in everyday life became well-established over the past half-century, helping to reveal speakers' ideas, attitudes and values (Cameron et al., 2009). Metaphor was defined by Aristotle as 'giving the thing a name that belongs to something else' (Gibbs, 1994, p. 210 ), the essence of which is echoed in the more developed characterisation of metaphor as a 'figure of speech in which a term or phrase with a literal meaning is applied to a different context in order to suggest a resemblance' (Sackmann, 1989, p. 465). Crucially, metaphors can be understood as more than simply words and expressions, instead reflecting a psychological essence which has been extensively explored through the lens of cognitive linguistics (Lakoff \& Johnson, 1980).

Spatial metaphors, specifically, have provided a conducive framework through which perceptions of environment, infrastructure and other physical or mental experiences can be understood, whether the emphasis is on broad concepts such as time (Moore, 2019) or the body politic (Rasmussen \& Brown, 2005), or specific interests such as feminist geographies (PriceChalita, 1994). In her comprehensive overview of the rise of spatial metaphors in contemporary social theory, Silber (1995) suggests a trend since the early 1980s in which the application of 
quasi-geographical expressions gained increasing currency. She singles out Pierre Bourdieu's work on fields and Harrison White's work on network theory as two spatially-strong metaphors, also listing less definitive conceptualisations, such as DiMaggio's 'multileveled architecture of culture', De Certeau's 'spaces' of resistance, and Habermas's 'public sphere' (Silber, 1995, pp. 343-344). Silber (1995, p. 327) makes an important distinction also applicable to this study, stating that her attention remains on the 'self-conscious, metaphorical usage of spatial constructs', rather than the commonsensical and 'orientational' categories permeating ordinary language and cognition. We follow that approach whereby, for example, Lackoff \& Johnson's work on orientational metaphors (such as $u p$, down) would be excluded, as we instead focus on more distinctive spatial imagery generated by Twitter users.

Spatial metaphors have also been used to capture attitudes towards digital environments (Maglio \& Matlock, 1998). Even a familiar expression such as 'web' is metaphorical, suggesting incremental growth and linked information over space and time (Matlock, Castro, Fleming, Gann, \& Maglio, 2014), an image common in early conceptualisations of the internet as a borderless digital realm, in which convergence grew and openness of ideas and exchanges prevailed. Arora (2014) documents the evolution of metaphors describing online space, highlighting the contemporary acceptance that virtual space is not a single, overarching structure, but is instead a plurality of networks shaped by various stakeholders. While digital spaces were once considered distinct and revolutionary, she describes how they became anchored to physical, real-world infrastructures, such as chatrooms, electronic frontiers, homepages, and information highways. Focusing on online spatial dimensions emphasises the importance of the underlying structure and design in shaping social action; Arora suggests that 'by capturing the shift in the nature and usage of metaphors for digital space, we can get a sense of the dominant concerns of our time' (2014, p. 13). Such an assessment underpins this study, in which the expressions used in discussions about 
blocklists provide the analytical context to help position users' understanding of the geographies, dynamics and affordances of contemporary social media interactions.

In addition, considering spatiality can be a useful tool to draw comparisons and links between online and offline activities (Lincoln \& Robards, 2014; Meek, 2012): young people’s social media profiles have been compared to bedrooms (Hodkinson, 2017; Lincoln \& Robards, 2014), raising issues of privacy, access, and displayed content, while Facebook has been seen as a scrapbook (Day Good, 2013). We propose one traditional spatial concept which may prove particularly appropriate in the architecture of online interactions: Osmond (1957), a psychiatrist, wrote of the distinction between socio-petal and socio-fugal physical spaces: the former is designed in such a way to encourage interaction between individuals (such as round tables), while the latter is a space in which people can, or try to, avoid one another (such as rows of seats in a waiting room). Although originally referencing hospital seating, it is a useful categorisation to bring to online spaces, especially amid potential shifts away from open, boundary-less communication flows to something more restricted through the application of blocklists or other filtering tools. Yet, we must also remember that there is no clear normative guide for such interactions: 'Sociofugal space is not necessarily bad, nor is sociopetal space universally good. What is desirable is flexibility and congruence between design and function so that there is a variety of spaces, and people can be involved or not, as the occasion and mood demand' (Hall, 1966, p. 110). This sense of autonomy and control is vital in explaining why Twitter users who sign up to blocklists decide to alter their own boundaries in such a way (Maréchal, 2016).

Building on this, it is useful to return to Arora's (2014) extensive work on spatial conceptualisation of Web 2.0 interfaces, which marked a turning point towards more individually driven online behaviours and a shift from the heavy distinction between space and the previously othered 'cyberspace' (Meek, 2012). Arora's work hinges on various conceptualisations of the 
public park, a leisure commons which, through various formats, faces challenges regarding access, privacy, design, regulation, and conflict. In theory, parks may be open to all, but in reality - like online spaces - they balance explicit rules and implied norms. Of specific interest in Arora's work is the 'walled garden' type of public space; she explains how walls 'can protect as well as suffocate', but such gardens can be viewed as 'a social response to containing the wilderness either by taming the undesirable or walling off from it' (2014, p. 57). Inherent to this, Arora argues, are struggles between private/public spheres and between inclusivity/exclusivity, raising concerns about the communality of such spaces, especially when divisions along social, racial, class, or sexuality lines become apparent. Nevertheless, Arora demonstrates how 'the fragmenting of groups can be antithetical to the public leisure commons, [but] it can also provide secure havens and intimate enclaves of sociality' (2014, p. 66); walled gardens with restricted access points may limit services but can protect users from outside negative influences, such as online viruses or spam. This indicates a key evolution in perception towards the internet, contrasting with previous notions of open, free-flowing spaces - such as an open 'protest park' - where the apparent lack of boundaries was advantageous.

Although underexplored in the literature, blocklists may fit in with this 'walled' infrastructure, especially given the tools' association with avoiding harassment (Geiger, 2016; Jhaver et al., 2018), resonating with narratives around the desire for safe spaces more generally (Clark-Parsons, 2018; Maliepaard, 2017; The Roestone Collective, 2014), or even 'sane spaces' to help preserve mental health (Wheatley \& Vatnoey 2020). Arora highlights the shift from open social networks like MySpace towards the quasi-public social grounds of Facebook, and suggests that social media sites are becoming increasingly compartmentalised through users' choices and behaviours, through which a 'systematic erosion of spontaneous social encounters and interactions' is apparent; we become more 'architecturally cocooned' as people seek security, trust 
and intimacy (Arora, 2014, p. 75). However, filtering on social networks has led to concerns about a lack of cross-cutting discussion and potential democratic ramifications (Heatherly, Lu, \& Lee, 2017; Sunstein, 2017). Yet to further understand these phenomena, it is useful to examine some of the forces associated with such segregation.

\section{Altered boundaries on social media}

Many observers highlight the algorithmic power of technology companies building targeted audience profiles (Bozdag, 2013; Pariser, 2011), while another perspective highlights the physical and mental actions of individuals in shaping their own digital spaces. This latter perspective can be tied to psychological and sociological concepts regarding homophily (McPherson, SmithLovin, \& Cook, 2001), selective exposure and cognitive dissonance (Stroud, 2010; Zillman \& Bryants, 1985) developed since the mid-20 $20^{\text {th }}$ century. Homophily captures the 'birds of a feather' trend in observations around (offline) social networks and the desire to connect, and retain links, with like-minded others (McPherson et al., 2001). Lazarsfeld and Merton (1954) distinguished between status homophily (tied to demographics and upbringing) and value homophily, the latter of which may resonate with polarising issues like abortion. Baron (1995) identifies the myside bias in thinking about abortion, suggesting advocates fail to seek out counterarguments that may undercut their claims. For some, the nature of the subject means they find it difficult to tolerate opposing opinions and seek out like-minded information (Luker, 1984), indicating a desire for cognitive consistency. In a digital environment, this desire may manifest in users taking action to secure and curate an environment of participants who align with their values; this may involve the formal exclusion of transgressors from such spaces to ensure more harmonious, and fewer hostile, interactions.

Crucial to the work on homophily is the choice individuals make regarding those with whom they interact, fundamentally linked to selective-tie dissolution and the ending of other 
relationships, thus potentially leading to homogenous personal networks (McPherson et al., 2001). When applied online, patterns are somewhat replicated, albeit perhaps through more diverse geographic networks. Colleoni et al (2014, p. 317) argue that political homophily is an important concept when studying online networks because 'it concerns the ability of digital media to support the formation of a public sphere, where a diversity of opinion and information can interact, or, conversely, to function as an echo chamber', reinforcing established perspectives. In studying Twitter, they found that as a social space, Twitter increased homophily, but as a space for news diffusion, there were more wide-ranging networks. This aligns with recent research allaying some concerns about filter bubbles, suggesting selective exposure online is less drastic than once thought (Borgesius et al., 2016). However, while serendipitous exchanges may prevail, tools like blocklists make filtering more deliberate and rigid, thus reinforcing existing perceptions and limiting diverse exposure which may, therefore, alter the previously fluid boundaries of online landscapes into something much more defined. Users who sign up to blocklist tools are clearly motivated, perhaps for a multitude of reasons, to establish clear physical and psychological barriers to their interactions; exploring how users articulate attitudes towards these actions can, therefore, reveal valuable insights towards personal, customised digital social media spaces.

\section{Methodology}

\section{Context and data}

The referendum on May 252018 asked the Irish public whether or not to repeal a 1983 constitutional amendment which effectively outlawed abortion in all circumstances; in late January 2018, a small group of pro-choice volunteers created the @ Repeal_Shield blocklist. All tweets containing the words 'Repeal Shield' $(n=38,063)$ were gathered via the Twitter API from the four-month period in which the blocklist was active; retweets $(n=15,379)$ were removed to 
avoid repetition, reducing the sample to 22,684 . This was further reduced as many Tweets had simply tagged the @Repeal_Shield account in conversations about the referendum more generally and/or would not contribute to any analysis of attitudes towards the blocklist. For that reason, the focus shifted to those Tweets in which the presence and use of the blocklist itself was discussed: to find those, all 22,684 tweets were read manually by one researcher, and approximately $10 \%$ $(n=2,247)$ were deemed to be of value for the next stage as they directly addressed the concept, presence and role of the blocklist in the run-up to the referendum.

\section{Metaphor analysis}

The socio-cognitive use of metaphors means that analyses can help researchers understand how individuals and groups express their experiences (Cameron et al., 2009), proving especially insightful for naturally occurring interactions (Coffey \& Atkinson, 1996), in this case Twitter exchanges. This brings attention to the study's research questions: RQ1: How are spatial metaphors used to talk about blocklists? RQ2: What do these metaphors tell us about attitudes towards Twitter's altered boundaries? As Coffey \& Atkinson explain, metaphor analysis can provide accounts from participants that 'justify, legitimate, excuse, and so on. They may be locating their own and others' actions or evaluations within particular frames of reference' (1996, p. 84). This is a particularly apt summary; in this study, by first establishing the dominant spatial metaphors, we can then use them to explore how users interpret this novel filtering architecture on Twitter and gain insight into contemporary attitudes towards segregated Twitter spaces.

A modification of Schmitt's (2005) work on metaphors was used to systematically explore the way in which the blocklist's presence was spatially considered, so it is useful to detail Schmitt's criteria for a metaphor: (i) a word or phrase which can be understood beyond its literal meaning; (ii) the literal meaning stems from an area of sensoric or cultural experience (source area); (iii) the source area is transferred to a second, often abstract area (target area). In terms of 
methodological process, Schmitt recommends initially identifying the target area as part of the planning process. In this study, the topic was spatial conceptualisations of Twitter and blocklists. Next, there was an unsystematic broad-based collection of background metaphors: this step is still preparatory, and Schmitt suggests lists of metaphorical expressions be drawn up from sources such as encyclopaedias, journals, etc, to create an initial overview of terms, rather than creating a list interpretively. In this case, spatial metaphors were found in existing literature (Silber, 1995), and other literal variations based on dictionary definitions and associated synonyms, such as 'place', 'edge', 'boundary', providing a comprehensive starting point.

The third step was a systematic analysis of a subgroup: This stage shifts attention to the corpus by extracting a sample to establish metaphors used by the Tweeters. Schmitt divides this stage into two processes: (i) the identification of metaphors and (ii) the reconstruction of metaphorical concepts. Firstly, in our search, a subsample $(n=540)$ of approximately $25 \%$ of the total Tweets were extracted. Any shifts in the overall discourse surrounding Repeal Shield at the start-, mid- and end-points of its existence were factored in by selecting three subsamples of 180 Tweets. Selecting consecutive tweets meant that from the 2,247 tweets, the following tweets (when ordered chronologically) were extracted: \#1-\#180,\#1000-\#1180,\#2000-\#2180. From these tweets, spatial metaphors were sought inductively and recorded. In some instances, it was simply one word, such as 'bubble' or 'barrier', while other expressions such as 'hiding behind' or 'keeping my timeline clean' also had spatial undertones. When the 540 Tweets were all read and metaphors recorded, the second stage of this step required synthesising collective metaphorical models, by linking specific metaphorical idioms/target areas to a much smaller number of four overarching metaphorical concepts (and two additional sub-concepts), until all idioms are clustered under one of the main concepts, thus providing the metaphorical framework of analysis. 
The remaining 1,707 Tweets in the sample were then read and coded to the relevant target area and metaphorical concept. Of course, not every Tweet contained any metaphorical element and, furthermore, not every metaphorical expression was coded and the results presented are the dominant images throughout the sample. One-off metaphors, which we considered conceptual outliers, are omitted from this analysis and metaphors had to be present in more than one Tweet to be presented here. As the study does not quantify the results in terms of demonstrating occurrence, we believe it was important that any metaphors described in this study were relatively common and used by more than one or two users to ensure a more accurate overview of dominant metaphors. Of course, there is a subjectivity problem inherent in any metaphor analysis and potential triangulation approaches could not be carried out in this case (Armstrong, Davis, \& Paulson, 2017). Nevertheless, every effort was made to ensure an accurate reading through detailed discussions between researchers and contextualising the metaphorical expressions within the original Tweets.

\section{Results \& Analysis}

The analysis revealed that the spatial conceptualisations of Twitter primarily relate to two central source domains: domestic/personal and battle/struggle. Thus, we identify two conceptual metaphors that are particularly relevant for how people talk and think about Twitter: TWITTER AS PERSONAL SPACE and TWITTER AS BATTLEFIELD. Untangling these two conceptual metaphors, we focus on three main target domains. The first one is Twitter itself, perceived as a space or a medium. The second is the interaction that constitutes the practice of this space, that is the talk or debate that Twitter users engage in. The third one is the Repeal Shield auto-blocker list which is the main topic in the material. We also identify two other conceptual metaphors specifically relevant to conceptualisations of space: MENTAL HEALTH AS A RESOURCE and BLOCKLISTS AS POLICING. These are not directly connected to the source domains 
domestic/personal and battle/struggle, but are relevant to how the actors talk and think about blocklists and to the dual relationship between new affordances (the introduction of autoblocklists) and spatial conceptualisations (how people talk and think about Twitter as a space).

\section{TWITTER AS PERSONAL SPACE}

The presence of various metaphors sourced from domestic life, referring to one's Twitter-feed as a house or dwelling, is central here, with some relating directly to conceptualisations of space. Blocklist users are described as living in an 'echo chamber' or 'igloo', and the blocklist is frequently referred to as a form of 'shelter', 'fence' or 'building'. The conceptual metaphor suggests that Twitter is a place where people are invited in or kept out; when people breach the blocklist, they are said to 'come out and play', 'step outside' or 'let people in', whilst the blocklist is referred to as 'shutting people out' or 'slamming the door' and users are 'locked in'. Several metaphors connect the target domain Twitter to domestic life by referencing household chores. People are 'cleaning' their feed, 'taking out the trash', 'sweeping it under the carpet', they get rid of the 'garbage', and claiming 'my [timeline] is less cluttered'.

1 Seriously you've changed my twitter experience. Everyone sign up and you'll live in a happy pro-choice twitasphere

2 If you want a civilised debate, get your own house in order first

3 So handy and makes your TL a better place

4 They live behind the safety of \#Repeal Shield! They're [sic!] own views are reinforced without question every day.

Table 1: Tweet extracts illustrating the metaphorical concept TWITTER AS PERSONAL SPACE

The conceptual metaphor TWITTER AS A PERSONAL SPACE is extended through users' words describing their own domain. Many positive descriptions of the blocklist suggest a key function of 
one's Twitter-feed is to serve as a safe and social space for resident(s). Blocklist users describe how their feed became 'a happier place', 'a nicer place', and 'a positive place'. One claims that 'Now I'm living in bliss', while others use 'serenity'. These praises bring forth connotations not of a public place, but of the private sphere, adding to conceptual understandings of Twitter as personal space.

We also find other conceptual metaphors relating to this personal space notion as various physical and bodily metaphors connect digital presence to a physical presence. Blocklist users are accused of 'burying their head in the sand', 'close their eyes and ears', and putting the 'fingers in their ears'. Certain Twitter users are said to be 'screaming' and their actions 'deafening'. Unwanted content is referred to as 'shit' and 'bile'. These kinds of metaphors are universal, and it would be more surprising if they were not regularly present in the material. Nevertheless, their presence indicates that users experience Twitter as a space capturing some kind of personal, almost physical presence.

\section{TWITTER AS BATTLEFIELD}

This second overarching conceptual metaphor connects different target domains to a source domain best described as battle or struggle, as the target domain (Twitter) is frequently described in battlefield terms. Users talk of 'middle ground', 'the other side' and 'hostile territory', employing military vocabulary like 'minefield', 'trenches', 'bunker', and 'refuge'. People engaging in the debate are said to have a 'weak position', they are 'out in the open', they 'occupy' territory, they 'duck down', 'take cover' or 'run back behind the shield'. The referendum is frequently referred to in terms of 'victory', 'defeat' or 'surrender'. The most salient metaphor describing interaction between the different sides is INTERACTION AS WAR. People of opposing viewpoints are referred to as 'enemies', blocklist users refer to opponents as 'raging hoards', their actions an 'onslaught' or 'full-frontal attack'. The military lingo surrounding the 
concept is rich: 'Invasion', 'attack', 'retreat', 'ring-fencing', 'fending off', 'bombarded by antichoice lies', 'the pro-lifers are disarmed'. Adding to this, references to participants in the debate frequently refer to them as 'heroes' or 'cowards' amid talk of 'honour', 'bravery', and 'resilience'. A clear case in point: 'You people are heroes. But the war isn't over yet'.

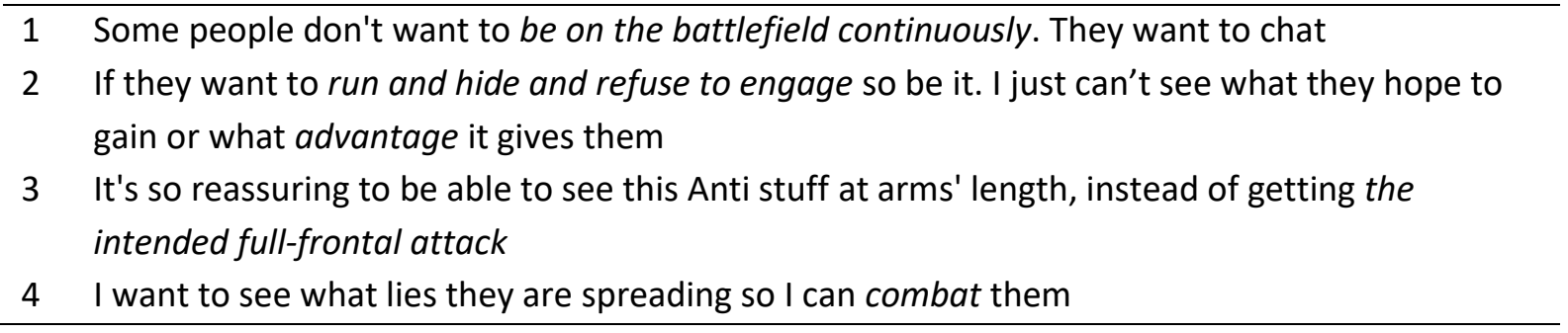

Table 2: Tweet extracts illustrating the metaphorical concept TWITTER AS BATTLEFIELD

It is in relation to this overarching BATTLEFIELD conceptual metaphor that the auto-blocker is most often understood. As the name 'Repeal Shield' suggests, the blocklist is conceptualised as a shield against attacks. The metaphor here is BLOCKLIST AS A WALL. The conceptual metaphor suggests that a blocklist separates and sections Twitter into delimited spaces, and both supporters and critics endorse this metaphor. On one hand, the blocklist users are accused of 'hiding behind the shield' or, more positively, are 'shielded' or take 'cover'. The blocklist is frequently referred to as a 'protective' means. On the other hand, those placed on the blocklist are seen to be 'fenced in' or 'placed behind' the virtual wall.

\section{MENTAL HEALTH AS RESOURCE}

The users of the auto-blocklist frequently talk about their own and others' mental health as motivation for signing up. While physical health often serves as a source domain for negative reactions, e.g. 'getting rid of the headaches', 'reducing blood pressure', mental health more often 
serves as target domain in a conceptual metaphor that can be articulated as MENTAL HEALTH AS RESOURCE. Users often talk about their own mental health as a resource that is 'drained', 'worn down', 'wasted', 'used up' or 'saved'. A typical comment is '[Repeal shield] is saving my sanity these days'. Another is the reassurance that 'you don't owe anyone your time or mental wellbeing'. One user's praise highlights how the list saves people from 'wasting their mental health by seeing thousands of abusive tweets'. More specifically, several users talk about mental health, or their 'sanity', as something to be saved and redeployed at more productive outcomes, such as canvassing or discussions with undecided voters.

1 [RS has] made Twitter a grand place to be again. Fuck the echo chamber nonsense: we need to mind our mental health

2 Do not engage with abusive Anti Choicers. It's not worth your energy or the damage to your mental health

3 The list has maintained my sanity lately

4 I know that the constant barrage of abuse and lies by those that want to deny me human rights would break me. The work they're doing keeps me sane!

\section{Table 3: Tweet extracts illustrating the metaphorical concept MENTAL HEALTH AS RESOURCE}

Furthermore, the conceptualisation of mental health as a resource suggests it must be protected. The referendum debate is frequently described as 'emotionally draining' or 'triggering', believed to have 'a lasting negative effect on mental health'. The conception of mental health dominating the material is thus not primarily drawn from a medical discourse, but is more often portrayed as a matter of safety, comfort and self-care. One's mental health is seemingly a resource that must be taken care of and protected. While the metaphor 'safe space' is mostly used ironically by blocklist critics, suggesting an avid coddling of one's own insecurities, the connection between safety and mental health is also frequent among the blocklist users. For instance, a common credo is that it is 
'important to keep safe'. The supporters of the blocklist urge each other to 'protect' their mental health and 'take care' of each other. The frequent link made between mental health, safety, and space, presupposes another conceptual metaphor: OFFENSIVE CONTENT AS (PHYSICAL) VIOLENCE. A clear example can be drawn from one blocklist supporter: 'I don't blame anyone feeling vulnerable blocking people to be safe'. Others praise the blocklist for 'keeping us safe'. One user expresses support for the blocklist users' 'right to protection'. Another claims that 'Help isn't a strong enough word. It really is protection from sheer aggression and hatred'. It is apparent in the way the supporters talk about mental health that they see the blocklist as a form of security measure, acting as a wall that keeps abusive, threatening elements out. Behind this wall is a safe and nurturing environment where the supporters' mental health is safeguarded.

\section{BLOCKLISTS AS POLICING}

Tweets criticising the blocklist frequently suggested the conceptual metaphor BLOCKLISTS AS POLICING. The blocklist is referred to as an attempt to control and suppress people of opposing beliefs; supporters and administrators are referred to in a manner suggesting illegitimate exercise of authority. Some users underline the controlling aspect of keeping a list by capitalising the letters: 'Immediate casting onto The List for insubordination'. Here it is also strongly suggested that the blocklist is a way to sanction unwanted behaviour. This is implied in the common reference to the blocklist as 'the blacklist': 'the spectacle of a "human rights man" here in the public, on a public forum, unable \& unwilling to condemn a fascist blacklist of 1,000 ideologically unapproved citizens should tell onlookers all they need to know about you'. This common connection between 'list' and 'citizens' that are 'put on' it, emphasises that 'the list' in this context acts as a synecdoche for surveillance. Others use the phrase 'ze list' as a rhetorical tactic invoking collective historical associations to the German secret police, or, as the quote above, explicitly describe it as 'fascist', 'Stasi', 'Orwellian' or 'McCarthyism'. One refers to supporters 
as 'a gang of whispering informers', while another suggests that the list is a way to 'roundup' political opponents. A similar conceptualisation of the list as abuse of power is that it invades people's privacy, functioning as a form of public humiliation as the list is visible to all.

Another frequent accusation tied to BLOCKLISTS AS POLICING is that the blocklist is a form of censorship. Some refer to being blocked as 'banned' or 'silenced', implying the blocklist administrators have assumed the authority to prohibit people. One user clearly places the blocklist in the context of power abuse by implying that it threatens basic political rights: 'I'm a free speech absolutist. Everyone should be free to agitate for whatever they happen to believe in. Without censorship, intimidation or repression'.

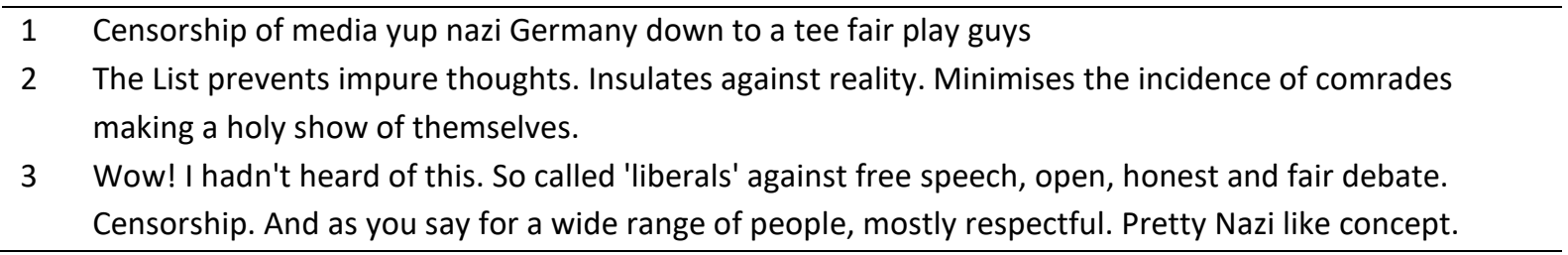

Table 4: Tweets illustrating the metaphorical concept BLOCKLISTS AS POLICING

What follows from this conceptual metaphor, and is particularly evident in the vocabulary surrounding it, is that the actors are placed in the role as citizens. A frequent claim is that the blocklist threatens people's civil and political rights, in particular, to privacy and freedom of speech. This suggests that the blocklist represents some form of power or authority comparable to the state power monopoly. It also suggests that Twitter, in line with this metaphor, is seen as a public - as opposed to a private - space. The conceptual metaphor of blocklists as a form of policing through surveillance and censorship assumes an understanding of these actions as public actions. 


\section{Discussion and Conclusion}

The analysis demonstrates how conceptualisations of social networks ebb and flow between both public and private spheres, as concerns about personal space appear out of sync with some users' normative expectations of a collective, discursive commons. Yet, intertwined through each metaphorical concept were patterns of harm, care, health, and safety, indicating these as essential considerations for those seeking to alter the structure of their online spaces. The Repeal Shield blocklist played a pivotal role in guarding the boundaries of such an area, and users who subscribed assert their agency by choosing to reside in such a 'safe space' or haven: they support the restricted accessibility, unconcerned by the fact that all parts of others' conversations are no longer visible. Conversely, in describing this same space, images were conjured up - through scathing, mocking language - of an 'echo chamber'. In this vision, residents are apparently naïve and sheltered from 'the truth', choosing not to interact with others, supposedly indicative of weak character. Although this safe space/echo chambers vernacular is well-established, blocklists provide a novel backdrop, raising new questions around how a technical tool has a role in shaping interactions and reinforcing visions of segregated spaces.

The disparity in how users perceive the affordances of auto-blocking demonstrates a fundamental discrepancy in how people believe online spaces should be constructed. For some who encountered problems online, this meant taking pro-active steps - such as subscribing to blocklists - to structurally alter their surroundings. In this way, we can again consider Osmond's (1957) work on the design of common spaces, and whether they encourage or deter interaction. Twitter's natural open-flow approach suggests its creators envisage a sociable, socio-petal space; conversely, blocklists indicate some users have a real desire to create socio-fugal spaces in which they feel more comfortable and peaceful. Although ideological issues of value homophily and affective polarisation may be factors, it appears the motivation for using blocklists is not based 
solely on a user's stance on abortion or 'myside bias' (Baron, 1995). Instead, it appears to run deeper into users' concern about their personal digital space: using the blocklist minimises harm and is repeatedly interpreted as a form of risk avoidance. This socio-fugal desire to create a nonviolent, non-harmful space was evident in both conceptualisations of Twitter as personal space and as a battlefield. The language and expressions used - such as military terms, coupled with concerns about wellbeing - illustrate how users interpret the perils they face online as tangible, grounding them in real-world threats to physical and mental wellbeing. This was highlighted clearly in the idea that interaction is war, a key reason for why some users wanted to avoid confrontational contact on Twitter. Although many highlighted the need for 'debate' and 'engagement' - as if that was the very essence of what Twitter should be about - others users' interpretation of the social network as a conflict zone meant they took an avoidance approach. This was especially apparent if visible material was deemed offensive, interpreted by many users as a form of violence.

The notion that women may face more abuse warrants attention, especially given the gendered dimension of previous blocklists (Jhaver et al., 2018; Maréchal, 2016): in this case, users highlighted how 'women especially' face attacks, females are 'targeted' and have 'different experiences' on Twitter. This was linked to feeling vulnerable, the need for safety, and avoiding physical and emotional distress. The gender element was likely exacerbated by the abortion subject, with women seeking refuge from anti-abortion material, especially those women who spoke out about miscarriages and abortions ('I'm incredibly grateful to @repeal_shield for making Twitter a less triggering and safer space for me while I'm pregnant again and navigate the anxiety involved after a loss'). The Repeal Shield administrators highlighted their desire to tackle this gendered element, saying they established the list in response to the 'silencing and intimidation of women by anti-abortion activists', and to help the referendum activists 'reclaim' their social 
media, as if it had become a colonised territory. They noted how the accounts they blocked rarely attacked men in the same way and highlighted the gratitude they received ('[We have] dozens of tweets from Irish women saying [that] the shield was incredibly helpful and makes Twitter a safer space'). Arora (2014) specifically raises the protection of women and children in her conceptualisation of the walled garden, and it is not difficult to see the overlap here as many users favoured the idea of restricted access. While this blocklist did not create a women-only space as seen elsewhere online (Clark-Parsons, 2018; Pruchniewska, 2019), it still draws on the idea of curating controlled areas in which women feel protected. Crucially, women themselves recognise the 'safe space' afforded to them by using such vernacular, despite the term being repeatedly mocked by sceptics. The fact the safe-space concept was repeatedly drawn on by both supporters and critics reinforces its role as an intrinsic notion in the deconstruction of contemporary digital spatialisation.

As highlighted, the interpretations of harm and risk online are articulated as material, suggesting that digital experiences are accepted as tangible parts of 'real' daily life.

Furthermore, through the use of bodily references (eyes, ears, head), we observed efforts to connect the digital presence to the physical presence, linking the online and offline. Yet, there were still clear distinctions evident throughout between virtual/real, raising important points regarding the extent to which digital spaces are still conceptualised as separate, and sometimes less valid. References were continually made to 'real life', the 'real world', or 'real campaigning' on the streets and at doors as something quite different to - and often more important than - Twitter activity. Linked to this were comments around the limitations of Twitter as a space ('Thankfully Twitter is not real life'/ 'Twitter is really an insignificant part of the world'). Despite this, there were responses to such attitudes, in which users pointed out that the problems encountered online warranted attention and should not be dismissed ('Have you seen the comments from those blocked 
accounts? Utterly vile. This is a real issue for real women \& needs to be discussed based on reality of lived experience'). These attitudes highlight that the harm and safety motivations for using the list at the core of many users' experiences do not remain contained in a digital sphere, nor is that digital sphere removed from the overall human experience. One further way to synthesise these perspectives on the 'reality' of the so-called Twittersphere is to accept that the harm felt is 'real', even if it is a form of psychological harm or violence interpreted by users as physical distress through the language of warfare. The blocklist then becomes the digital affordance providing a form of authentic, tangible relief, acting as a means to preserve the mental health resources needed to survive battle - but that relief has limitations. This meant the tool was in no way replicable in any physical sense (despite some users longing for 'an offline Repeal Shield'). Therefore, we can see the Twitter environment as both the cause and solution to some problems users faced online, affecting both their digital and material experiences.

Elsewhere in this consideration of 'reality', some critics also suggested that the personal, domestic areas that blocklist users were creating (igloo, cocoon, bunker), were somehow removed from reality. Crucially, this was not because it was a digital environment, but because it was designed in a way that provided shielding or shelter which meant users were unaware of their true surroundings ('[RS users] are gonna find the real world a scary place!'). The blocklist was interpreted in this context as problematic as it suggested the sheltering dimension was creating inauthentic experiences, yet these commentators seemingly accepted that the 'truth' and 'reality' was ever-present online.

Finally, the often-cited expectation that Twitter is a civic forum was the backdrop against which many positioned the so-called interference of the blocklist. Users repeatedly tied their criticism of the list to its role in curbing conversation and the curation of delimited spaces, and we again see concerns about how blocklists supposedly transgress the 'rules' on online engagement 
(Gosse \& O’Meara, 2018). The desire for open interaction resonates with the earlier conceptualisations of cyberspace as free-flowing and open, a paradigm which appears to be shifting as customisable tools and filtering develop. Whether critically comparing the blocklist to illegitimate policing which restricts citizen Tweeters, endorsing its role as a helpful moderator, or even simply acknowledging its construction as a barrier to interaction, the metaphor of Repeal Shield as a dividing force was endorsed by all sides, as was the conflict metaphor. The warfare language evident here is a manifestation of Twitter as a space for battles to be fought and its use is unsurprising in a political context in which there is something tangible in contention. As part of this political campaign, some users felt they needed sanity and strength and that risk avoidance was the solution, while others sought conflict to help achieve victory. Either way, the conceptualisation of the blocklist's role is inherently linked to spatial conceptualisations in which autoblockers are seen as a dividing force that facilitates isolation, be that in a positive or negative way.

The Repeal Shield blocklist and the Irish case study may not be generalisable given the niche interest of a national referendum in a country with a population of fewer than five million people, only a tiny fraction of whom would have even been aware of such a blocklist.

Furthermore, the polarisation associated with abortion - and the binary nature of referendums may have exacerbated divisions which manifested in heightened tension between sides and brought the sense of conflict to the fore. Therefore, some findings may not be applicable to all cases, but still provide valuable insight into this understudied subject area. For those looking at blocklists in future, it is important to delve into as many case studies as possible from different contexts, while a gap also exists in empirical knowledge about the motivations and rationale of those who establish and administer blocklists. It would also be beneficial to tie blocklist use to broader research exploring motivations about customising boundaries online and the desire (or lack thereof) to connect with others. 


\section{References}

Armstrong SL, Davis HS and Paulson EJ (2017) The Subjectivity Problem: Improving Triangulation Approaches in Metaphor Analysis Studies. International Journal of Qualitative Methods 10(2): 151-163. DOI: 10.1177/160940691101000204

Arora, P. (2014). The Leisure Commons: A Spatial History of Web 2.0. New York: Routledge.

Auerbach, D. (2015). Beware the Blocklists. Retrieved July 24, 2018, from http://www.slate.com/articles/technology/bitwise/2015/08/twitter_blocklists_they_can_stop_ harassment_and_they_can_create_entirely.html?via=gdpr-consent

Baron, J. (1995). Myside bias in thinking about abortion from the National Sci-ence Foundation (SES-8509807 and SES-8809299). I thank Nigel Harvey for suggest-ing the last experiment. Address correspondence to. Thinking and Reasoning, 1(3), 221-235.

Benesch, S. (2017). Civil Society Puts a Hand on the Wheel: Diverse Responses to Harmful Speech. Perspectives on Harmful Speech Online: A Collection of Essays, (August), 42-43.

Borgesius, F. J. Z., Trilling, D., Möller, J., Bodo, B., De Vreese, C. H., \& Helberger, N. (2016). Should we worry about filter bubbles? Internet Policy Review, 5(1), 1-16.

Bozdag, E. (2013). Bias in algorithmic filtering and personalization. Ethics and Information Technology, 15(3), 209-227.

Cameron, L., Maslen, R., Todd, Z., Maule, J., Stratton, P., \& Stanley, N. (2009). The discourse dynamics approach to metaphhor and metaphor-led discourse analysis. Metaphor and Symbol, 24(2), 63-89.

Clark-Parsons, R. (2018). Building a digital Girl Army: The cultivation of feminist safe spaces online. New Media and Society, 20(6), 2125-2144.

Coffey, A., \& Atkinson, P. (1996). Making Sense of Qualitative Data. Thousand Oaks, CA: SAGE Publications.

Colleoni, E., Rozza, A., \& Arvidsson, A. (2014). Echo Chamber or Public Sphere? Predicting Political Orientation and Measuring Political Homophily in Twitter Using Big Data. Journal of Communication, 64(2), 317-332.

Day Good, K. (2013). From scrapbook to Facebook: A history of personal media assemblage and archives. New Media and Society, 15(4), 557-573.

Geiger, R. S. (2016). Bot-based collective blocklists in Twitter: the counterpublic moderation of harassment in a networked public space. Information Communication and Society, 19, 787803.

Gibbs, R. W. (1994). The Poetics of Mind: Figurative Thought, Language, and Understanding. Cambridge: Cambridge University Press.

Golbeck, J., Gnanasekaran, R. K., Gunasekaran, R. R., Hoffman, K. M., Hottle, J., Jienjitlert, V., 
... Gergory, Q. (2017). A Large Labeled Corpus for Online Harassment Research.

Proceedings of the 2017 ACM on Web Science Conference - WebSci '17, 229-233.

Gosse, C. E., \& O’Meara, V. J. (2018). “Blockbotting Dissent”:Publics , Counterpublics, and Algorithmic Public Sphere(s). Stream: Inspiring Critical Thought, 10(1), 3-11.

Hall, E. T. (1966). The hidden dimension. New York: Doubleday \& Co.

Heatherly, K. A., Lu, Y., \& Lee, J. K. (2017). Filtering out the other side? Cross-cutting and likeminded discussions on social networking sites. New Media and Society, 19(8), 1271-1289.

Hodkinson, P. (2017). Bedrooms and beyond: Youth, identity and privacy on social network sites. New Media and Society, 19(2), 272-288.

Jhaver, S., Ghoshal, S., Bruckman, A., \& Gilbert, E. (2018). Online Harassment and Content Moderation: The Case of Blocklists. ACM Trans. Comput.-Hum. Interact, 25(2), 1-33.

Lakoff, G., \& Johnson, M. (1980). Metaphors We Live By. Chicago: The University of Chicago Press.

Lazarsfeld PF and Merton RK (1954) Friendship as a social process: A substantive and methodological analysis. In: Berger M, Abel T, and Page CH (eds) Freedom and Control in Modern Society. New York: Van Nostrand, pp. 18-66.

Lincoln, S., \& Robards, B. (2014). Being strategic and taking control: Bedrooms, social network sites and the narratives of growing up. New Media and Society, 18(6), 927-943.

Luker, K. (1984). Abortion and the Politics of Motherhood. Berkeley: University of California Press.

Maglio, P. P., \& Matlock, T. (1998). Metaphors we surf the web by. Workshop on Personal and Social Navigation in Information Space, (1980), 1-9.

Maliepaard, E. (2017). Bisexual Safe Space(s) on the Internet: Analysis of an Online Forum for Bisexuals. Tijdschrift Voor Economische En Sociale Geografie, 108(3), 318-330.

Maréchal, N. (2016). When bots tweet: Toward a normative framework for bots on social networking stes. International Journal of Communication, 10(June), 5022-5031.

Matlock, T., Castro, S. C., Fleming, M., Gann, T. M., \& Maglio, P. P. (2014). Spatial Metaphors of Web Use. Spatial Cognition and Computation, 14(4), 306-320.

McPherson, M., Smith-Lovin, L., \& Cook, J. M. (2001). Birds of a Feather: Homophily in Social Networks. Annual Review of Sociology, 27(1), 415-444.

Meek, D. (2012). YouTube and Social Movements: A Phenomenological Analysis of Participation, Events and Cyberplace. Antipode, 44(4), 1429-1448.

Moore, K. E. (2019). Introduction Talking about time as if it were space. In Spatial language of time. 
Osmond, H. (1957). Function as the Basis of Psychiatric Ward Design. Mental Hospitals, April, $23-27$.

Papacharissi, Z. (2009). The virtual geographies of social networks: A comparative analysis of Facebook, LinkedIn and ASmallWorld. New Media and Society, 11(1-2), 199-220.

Pariser, E. (2011). The Filter Bubble: What the Internet is Hiding from You. London: Penguin.

Poulos, J. (2015). How Block Bot could save the Internet. Retrieved June 6, 2019, from https://www.thedailybeast.com/how-block-bot-could-save-the-internet

Price-Chalita, P. (1994). Spatial Metaphor and the Politics of Empowerment: Mapping a Place for Feminism and Postmodernism in Geography? Antipodee, 26(3), 236-254.

Pruchniewska U (2019) “A group that's just women for women”: Feminist affordances of private Facebook groups for professionals. New Media \& Society: 146144481882249. DOI: $10.1177 / 1461444818822490$

Rasmussen, C., \& Brown, M. (2005). The body politic as spatial metaphor. Citizenship Studies, 9(5), 469-484.

Sackmann, S. (1989). The Role of Metaphors in Organization Transformation. Human Relations, 42(6), 463-485.

Schmitt, R. (2005). Systematic Metaphor Analysis as a Method of Qualitative Research. The Qualitative Report, 10(2), 358-394.

Silber, I. F. (1995). Space, fields, boundaries: The rise of spatial metaphors in contemporary sociological theory. Social Research, 62(2), 323-355.

Stroud, N. J. (2010). Polarization and partisan selective exposure. Journal of Communication, 60, $556-576$.

Sunstein, C. R. (2017). \#republic: Divided democracy in the age of social media. Princeton, New Jersey: Princeton University Press.

The Roestone Collective. (2014). Safe Space : Towards a Reconceptualization. Antipode, 46(5), $1346-1365$.

Wheatley D and Vatnoey E (2020) 'It's Twitter, a bear pit, not a debating society': A qualitative analysis of contrasting attitudes towards social media blocklists. New Media and Society 22(1): 5-25. DOI: 10.1177/1461444819858278.

Zillman, D., \& Bryants, J. (1985). Selective Exposure to Communication. (D. Zillman \& J. Bryant, Eds.). London: Routledge. 